\title{
Scaling of the Thue-Morse Diffraction Measure Acta Physica Polonica A 125, 431 (2014), ERRATUM
}

\author{
M. BAAKE ${ }^{a}$, U. GRIMM ${ }^{b}$ AND J. NiLSSON ${ }^{a}$ \\ ${ }^{a}$ Fakultät für Mathematik, Universität Bielefeld, Postfach 100131, 33501 Bielefeld, Germany \\ ${ }^{b}$ Department of Mathematics and Statistics, The Open University, Walton Hall, Milton Keynes MK7 6AA, UK \\ In the proofreading stage we overlooked a number of typographical errors. (On-line version is correct, this \\ erratum applies to printed version only.)
}

original DOI: 10.12693/APhysPolA.125.431

actual DOI: 10.12693/APhysPolA.129.1263

PACS/topics: 99.10.Cd

Under point 2 of Proposition 1, the last line must read $\lim _{N \rightarrow \infty} \frac{1}{N} \operatorname{card}\left(\left\{x_{n} \mid n \leq N\right\} \cap[a, b)\right)=b-a$.

Under points 3 and 4 of the same proposition, it must read $\frac{1}{N} \sum_{n=1}^{N}$ rather than $\frac{1}{n} \sum_{n=1}^{n}$. The same correction applies to the last equation in the right column of page 431 .

Furthermore, the first formula under Case B (on page 432) must begin as

$$
\beta(k)=\lim _{N \rightarrow \infty} \frac{1}{N} \sum_{n=0}^{N-1} \frac{\log \left(1-\cos \left(2^{n+1} \pi k\right)\right)}{\log (2)} .
$$

More importantly, our argument for Case B on the basis of uniform distribution modulo 1 of the sequence $\left(2^{n} x\right)_{n \in \mathbb{N}}$ for almost all $x \in \mathbb{R}$ is incomplete, because the function $f$ defined by $f(x)=\log (1-\cos (2 \pi x))$ is only Riemann integrable on $[0,1]$ in the generalised sense (meaning that it is an improper integral), which is insufficient here. However, $f$ is properly integrable on $[0,1]$ in the Lebesgue sense and has an obvious 1-periodic extension to $\mathbb{R}$.
Consequently, rather than employing uniform distribution, one can argue with the dynamical system defined by the map $T$ of the unit interval $[0,1]$ into itself, given by $x \mapsto 2 x \bmod 1$. It is well-known that $T$ leaves Lebesgue measure invariant and is ergodic relative to it, so that the Birkhoff sums satisfy

$$
\lim _{N \rightarrow \infty} \frac{1}{N} \sum_{n=0}^{N-1} f\left(T^{n}(k)\right)=\int_{0}^{1} f(x) \mathrm{d} x
$$

for Lebesgue almost every $k \in \mathbb{R}$ by an application of the ergodic theorem.

This still gives the result of Case B for almost all wave numbers $k \in \mathbb{R}$, though it might be that they differ from the wave numbers with uniform distribution of $\left(2^{n} x\right)_{n \in \mathbb{N}}$ on a null set. This has no further consequence on the analysis presented in paper.

\section{Acknowledgments}

We thank Gerhard Keller, Marc Keßeböhmer and Tanja Schindler for helpful comments and discussions. 